\title{
Diagnosis of heart failure with preserved ejection fraction. Not seeing the forest for the trees or a step to precision medicine?
}

\author{
Grigorios Korosoglou $^{1,2} \cdot$ Markus Haass $^{3}$
}

Published online: 29 May 2021

(c) The Author(s), under exclusive licence to Springer Nature B.V. 2021

In this issue of the journal, Shono et al. [1] report on the ability of leg-positive pressure (LPP) stress echocardiography to differentiate between patients with heart failure and preserved ejection fraction (HFpEF) from physiologic aging of the myocardium. Twenty-five patients with HFpEF and 77 healthy controls, including 27 young (20-40 years), 25 middle-aged ( $40-65$ years) and 25 elderly ( $>65$ years) were evaluated. The echocardiography protocol included measures of (i) the left ventricular (LV) mass-to-volume ratio, as a marker of LV remodeling, (ii) the E/é ratio, as a marker of the LV filling pressure, and (iii) forward stroke volume using pulsed-wave Doppler. Preload stress echocardiography was performed using commercially available LPP equipment in all subjects, obviating the need for physical stress testing, and allowing the calculation of the E/é ratio and stroke volumes during this maneuver. The authors found that progressive concentric LV remodeling is observed with increasing age in healthy subjects, being accompanied by increased LV stiffness. In addition, during preload LPP mediated stress, the E/é ratio remained unchanged with younger patients but increased significantly with middle-aged and elderly subjects and in patients with HFpEF. Forward stroke volume on the other hand, significantly increased in all control subjects but failed to increase in HFpEF. Using multivariable analysis, markers of LV mass-to-volume ratio, E/é ratio and LV stroke volume were independently associated with HFpEF versus physiologic myocardial aging, with accuracy rates of $\sim 85 \%, \sim 90 \%$ and $73 \%$, respectively for the identification of patients with HFpEF. Furthermore, LV mass index

Grigorios Korosoglou

gkorosoglou@hotmail.com

1 GRN Hospital Weinheim, Department of Cardiology, Vascular Medicine \& Pneumology, Weinheim, Germany

2 Cardiac Imaging Center Weinheim, Hector Foundation, Weinheim, Germany

3 Department of Cardiology, Theresienkrankenhaus, Mannheim, Germany and changes in forward stroke volume exhibited incremental value in clinical models for the differentiation between patients with HFpEF and similar aged control subjects.

The study of Shono et al. [1] makes an important step towards better understanding the underlying pathophysiology of HFpEF; however, some specific points need to be considered when interpreting the results of this study. Thus, a relatively small number of patients with HFpEF and similarly aged healthy controls was analyzed at a single time point, whereas follow-up data are not provided. In addition, the protocol did not include cardiac magnetic resonance (CMR) or laboratory markers, including light chain assays, so that some of patients classified as HFpEF may have exhibited other specific underlying disorders. Furthermore, echocardiography or CMR based strain measures were not available, which is a limitation since patients with HFpEF usually exhibit impaired myocardial strain in the presence of normal EF. Finally, therapeutic implications are currently limited in patients with HFpEF, which limits the translation of these findings into the clinical realm from a therapeutic point of view. In addition, the findings provided by Shono et al. need to be considered in the context of the current approaches for the diagnostic classification and risk stratification of patients with heart failure, giving special attention to HFpEF [2]. Thus, HFpEF currently accounts for more than half of patients with heart failure and its prevalence continuously rises relative to those with heart failure with reduced ejection fraction (HFrEF) due to ischemic or dilated cardiomyopathies [3]. In addition, the mortality rate of HFpEF patients is similar to age matched HFrEF patients. However, the mode of death in HFrEF is only in approximately two-thirds due to cardiovascular reasons [4]. Recent large-scale trials suggested that the heart is just one of the organs affected in HFpEF. Thus, this disease needs to be considered as a systemic disorder, which may also affect the pulmonary system, the neurohumoral and the renal axis. Therefore, in such patients, attention is necessary to avoid not seeing the forest for the trees. In addition, 
patients with HFpEF are predominantly female and often exhibit advanced age, obesity, arterial hypertension, renal failure, and sleep apnea [5, 6]. Such comorbidities may initiate chronic systemic inflammation, which can be detected in form of high plasma levels of inflammatory markers in patients with HFpEF [7]. This may affect myocardial remodeling and stiffness by endothelial dysfunction, infiltration of the myocardium by activated macrophages, ultimately causing increased myocardial stiffness due to interstitial fibrosis [8].

Despite advances in the diagnostic work-up of patients with heart failure, HFpEF is still considered as more or less a 'diagnosis of exclusion'. Thus, once an ejection fraction of $>50 \%$ has been documented in the presence of heart failure symptoms, cardiac imaging techniques focus on determining, if there is objective evidence of structural or functional myocardial disorders. Using standard echocardiography, LV diastolic dysfunction, left atrial dilatation and increased pulmonary artery pressure are common findings in patients with HFpEF [9]. Traditionally, right heart catheterization used to be the gold standard for the assessment of LV filling pressures. However, this technique is invasive and not suitable for serial evaluations. Therefore, Doppler transmitral flow patterns, pulsed tissue Doppler of the mitral anulus and the left atrial volume index are currently recommended for the estimation of LV filling pressures [10]. In addition, the mitral E velocity by PW Doppler can be divided by the é, derived from tissue Doppler, thus correcting for the influence of relaxation. The resultant E/é ratio has been shown to be strongly associated to the mean pulmonary capillary wedge pressure, as a surrogate marker of the LV filling pressure [11]. Cardiac magnetic resonance (CMR) on the other hand, is not as widely available as echocardiography but is considered as the gold standard for measuring LV mass, volumes, and the LV concentricity index, being free of geometric assumptions made by $2 \mathrm{D}$ echo and unaffected by acoustic windows [12]. In addition, CMR allows for tissue characterization using late gadolinium enhancement (LGE) for the detection of focal, diffuse, or patchy ischemic or non-ischemic scar patterns, whereas native T1 mapping techniques and extracellular volume (ECV) measures aid the detection of diffuse interstitial fibrosis [13]. Importantly, CMR can provide valuable information on the differential diagnosis of specific cardiomyopathies such as hypertrophic cardiomyopathy or infiltrative cardiomyopathies, such as cardiac amyloidosis, sarcoidosis, and the Anderson Fabry disease. This is crucial since patients with these disorders may strongly benefit from specific therapeutic algorithms. In this context, recent CMR studies reported to identify new specific pathologies such as ischemic heart disease, hypertrophic cardiomyopathy, or constrictive pericarditis in $27 \%$ of patients, initially classified as HFpEF by clinical and echocardiographic assessment [14]. In the same line, patients with cardiac amyloidosis may have been misclassified as HFpEF in several previous clinical trials, which did not include CMR, light chain assays and technetium-labeled cardiac scintigraphy by investigational protocols. Thus, the effectiveness of pharmacotherapy in HFpEF may have been underestimated in previous trials in this context [15].

The assessment of myocardial deformation using feature tracking or strain-encoded MR (SENC) has been thoroughly investigated in previous trials in patients with ischemic heart disease and heart failure [16-18]. In contrast to feature tracking, SENC allows for more reproducible and comprehensive assessment of myocardial strain both on segmental and global level [19]. In this regard, we recently demonstrated that assessment of the percentage of 'normal' myocardium by SENC can reclassify patients at risk for heart failure due to arterial hypertension or diabetes, i.e., at stage $\mathrm{A}$ based on ACC/AHA guidelines [20] to stage B, i.e., indicating subclinical LV dysfunction [17]. Such patients exhibit diminished myocardial strain in the absence of structural or functional abnormalities by echocardiography and in most cases have normal ejection-fraction, compatible with HFpEF. Despite preserved ejection-fraction however, the percentage of impaired normal myocardium predicted poor outcome at follow-up, which translated in increased all-cause mortality and hospitalizations due to heart failure symptoms [17]. This may bear tremendous socioeconomic implications, since the percentage of normal myocardium may aid the identification of several millions of presumable healthy individuals at risk for overt heart failure, who may profit from early risk factor control, lifestyle modifications and pharmacologic treatment.

Despite the limitations of the trial conducted by Shono et al. [1] and the absence of CMR and strain analyses, the study makes an important step to precision medicine aiding the accurate diagnosis of HFpEF by a relatively simple and practical approach. Especially LPP stress echocardiography provides the assessment of dynamic changes in terms of forward stroke volume and LV diastolic filling pressures. LPP is associated with lower time spent than exercise echocardiography and can therefore easily be integrated into daily clinical practice. In addition, although currently no specific therapy apart from symptomatic administration of diuretics has been shown to improve the clinical course of patients with HFpEF, major randomized trials are underway, which evaluate the role of Sodium-glucose cotransporter 2 (SGLT2) inhibitors, such as Dapagliflozin and Empagliflozin in HFpEF patients (clinicaltrials.gov/NCT03619213 and NCT03057951). Interestingly, recent studies have already shown that the SGLT2 inhibitor empagliflozin reversed myocardial hypertrophy and remodeling and reduced ECV, a marker of myocardial fibrosis in patients with coronary artery disease and type 2 diabetes mellitus [21]. Thus, SGLT2 inhibitors, which have recently revolutionized the 
treatment of HFrEF, may also prove beneficial for the treatment of HFpEF in future studies.

\section{References}

1. Shono-A, Matsumoto-K, Yamada-N et al (2021) Accelerated aging" of the heart as heart failure with preserved ejection fraction $\sim$ Analysis using leg-positive pressure stress echocardiography. Int $\mathrm{J}$ Cardiovasc Imaging. In press

2. Ponikowski P, Voors AA, Anker SD et al (2016) 2016 ESC Guidelines for the diagnosis and treatment of acute and chronic heart failure: the Task Force for the diagnosis and treatment of acute and chronic heart failure of the European Society of Cardiology (ESC). Developed with the special contribution of the Heart Failure Association (HFA) of the ESC. Eur Heart J 37:2129-2200

3. Borlaug BA (2015) Heart failure with preserved ejection fraction. In: Baliga RR, Haas GJ (eds) Management of heart failure. Springer, Berlin

4. Zile MR, Gaasch WH, Anand IS et al (2010) Mode of death in patients with heart failure and a preserved ejection fraction: results form the Irbesartan in Heart Failure With Preserved Ejection Fraction Study (I-Preserve) trial. Circulation 121:1393-1405

5. Haass M, Kitzman DW, Anand IS et al (2011) Body mass index and adverse cardiovascular outcomes in heart failure patients with preserved ejection fraction/clinical perspective. Circ Heart Fail 4:324-331

6. Dhingra A, Garg A, Kaur S et al (2014) Epidemiology of heart failure with preserved ejection fraction. Curr Heart Fail Rep 11:354-365

7. Sanders-van Wijk S, van Empel V et al (2015) Circulating biomarkers of distinct pathophysiological pathways in heart failure with preserved vs. reduced left ventricular ejection fraction. Eur J Heart Fail 17:1006-1014

8. Westermann D, Lindner D, Kasner M et al (2011) Cardiac inflammation contributes to changes in the extracellular matrix in patients with heart failure and normal ejection fraction. Circ Heart Fail 4:44-52

9. Redfield MM (2016) Heart failure with preserved ejection fraction. N Engl J Med 375:1868-1877

10. Chacon-Portillo MA, Acharya T, Janardhanan R (2021) Imaging in heart failure with preserved ejection fraction: insights into echocardiography and cardiac magnetic resonance imaging. Rev Cardiovasc Med 22:11-24
11. Nishimura RA, Appleton CP, Miller FA et al (2000) Clinical utility of Doppler echocardiography and tissue Doppler imaging in the estimation of left ventricular filling pressures. Circulation 102:1788-1794

12. Korosoglou G, Giusca S, Gitsioudis G et al (2014) Cardiac magnetic resonance and computed tomography angiography for clinical imaging of stable coronary artery disease. Diagnostic classification and risk stratification. Front Physiol 5:291

13. Taylor AJ, Salerno M, Dharmakumar R et al (2016) T1 mapping. JACC: Cardiovasc Imaging 9:67-81

14. Kanagala P, Cheng ASH, Singh A et al (2018) Diagnostic and prognostic utility of cardiovascular magnetic resonance imaging in heart failure with preserved ejection fraction-implications for clinical trials. J Cardiovasc Magn Reson 20:4

15. Oghina S, Bougouin W, Bézard M et al (2021) The impact of patients with cardiac amyloidosis in HFpEF trials. JACC Heart Fail 9:169-178

16. Korosoglou G, Giusca S, Hofmann NP et al (2019) Strain-encoded magnetic resonance: a method for the assessment of myocardial deformation. ESC Heart Fail 6:584-602

17. Korosoglou G, Giusca S, Montenbruck M et al (2021) Fast strainencoded cardiac magnetic resonance for diagnostic classification and risk stratification of heart failure patients. JACC Cardiovasc Imaging. https://doi.org/10.1016/j.jcmg.2020.10.024

18. Ochs MM, Kajzar I, Salatzki J et al (2021) Hyperventilation/ breath-hold maneuver to detect myocardial ischemia by strainencoded CMR: diagnostic accuracy of a needle-free stress protocol. JACC Cardiovasc Imaging. https://doi.org/10.1016/j.jcmg. 2021.02.022

19. Giusca S, Korosoglou G, Zieschang V et al (2018) Reproducibility study on myocardial strain assessment using fast-SENC cardiac magnetic resonance imaging. Sci Rep 8:14100

20. Ammar KA, Jacobsen SJ, Mahoney DW et al (2007) Prevalence and prognostic significance of heart failure stages: application of the American College of Cardiology/American Heart Association heart failure staging criteria in the community. Circulation 115:1563-1570

21. Mason T, Coelho-Filho OR, Verma S et al (2021) Empagliflozin reduces myocardial extracellular volume in patients with type 2 diabetes and coronary artery disease. JACC Cardiovasc Imaging. https://doi.org/10.1016/j.jcmg.2020.10.017

Publisher's Note Springer Nature remains neutral with regard to jurisdictional claims in published maps and institutional affiliations. 